\title{
Growth of Preferentially Oriented Aluminum Single Crystals
}

\author{
Theodore H. Orem
}

\begin{abstract}
Monocrystalline aluminum specimens having both a preferential orientation and a specified cross-sectional shape are very difficult to produce. Such specimens must be grown in a vertical furnace, a condition which, while permitting the choice of cross-sectional shape in the specimen, makes it extremely difficult to obtain specimens of preferred orientation. It is fairly easy to grow preferentially oriented monocrystalline aluminum specimens when a horizontal furnace is used, but the use of such a furnace limits the choice of cross-sectional shapes in the finished crystal. Crystals of circular cross-sectional shape are practically impossible to grow in a horizontal furnace.

The method deseribed herein can be used to grow monocrystalline aluminum crystals with any desired orientation or cross-sectional shape.
\end{abstract}

\section{Introduction}

Single crystals of metals are widely used in fundamental research on metals. They are grown by several different methods, one of the most popular being the Bridgman method of slow cooling from the melt. ${ }^{1}$ The method consists essentially in causing a molten mass of metal to slowly enter a zone maintained at a temperature below the melting point of the metal. As this occurs, the small portion of the metal that enters the cool zone first, freezes. The remainder of the metal subsequently entering the cool zone will then solidify in accordance with the orientation of the initially solidified portion of the melt, if the rate of passage from the molten zone to the cool zone is properly controlled.

The most popular way of growing single crystals by this method is by the lowering of the molten metal from a hot zone in a vertical furnace into a zone whose temperature is below that of the melting point of the metal, or by raising a furnace over a stationary molten mass of metal so as to cause the metal to freeze first at its lowest point.

A variation of the Bridgman method makes use of a horizontal-type furnace in which the molten metal, in a boat-type container, is pulled slowly from the molten zone into a cool zone. ${ }^{2}$

It is often desirable that single crystals have their axes preferentially oriented. This is accomplished by a method generally known as "seeding." It consists in attaching a small crystal of the metal to the sample that is to be grown as a single crystal, in such a manner that the crystallographic orientation of the "seed" is parallel to the crystallographic orientation desired in the single crystal to be produced. One adjusts the heating conditions so that the entire charge, with the exception of a small portion of the seed crystal, is molten. By properly controlling the rate of withdrawal of the melt from the hot zone, it is possible to cause the molten metal to solidify as a single crystal whose crystallographic orientation is identical with that of the seed.

\footnotetext{
${ }_{1}$ P. W. Bridgman, Proc. Am. Acad. Arts Sci. 60, 305 (1935).
}

2 B. Chalmers, Can. J. Phys. 31, 132 (1953).
When grown by means of a vertical furnace it is possible to produce single crystals of any desired cross-sectional shape. It is, however, very difficult to grow single crystals of preferred orientation in a vertical furnace because of difficulty in attaching and partially melting the seed crystal. It is relatively more simple to attach and partially melt a seed crystal in order to grow preferentially oriented single crystals in a horizontal furnace. In such a furnace, however, it is impossible to grow single crystals of a desired cross-sectional shape.

Described herein is a method for growing single crystals of aluminum ( $\mathrm{mp} 660^{\circ} \mathrm{C}$ ) of any desired crystallographic orientation, using a vertical furnace, in order that crystals of any desired cross-sectional shape may be produced.

\section{Materials and Description}

The apparatus required for the production of aluminum single crystals of preferred axial orientation consists of a vertical tube furnace, a means of controlling and recording temperatures within the furnace, a means of conducting heat away from an area within the furnace, the usual crucibles used for melting metals, and a special crucible for melting the seed crystal. The crucibles are machined from graphite.

Figure 1 is a diagrammatic sketch of a furnace showing the equipment used for the seeding of aluminum single crystals grown by the Bridgman method of slow cooling from the melt. Figure 2 shows longitudinal sections of the main crucible, the seed-melting crucible and the device used for conducting heat away from the seed crucible. This latter device is the means by which it is possible to melt just a portion of the seed. Also shown is a second seed crucible whose function will be explained later.

In addition to the above, there is also required a quantity of seed crystals. These are aluminum single crystals, $1 / 4$ in. in diameter by $2 \frac{1}{2}$ in. long, which are grown in graphite crucibles by the Bridgman method. These are easy to grow and can be 


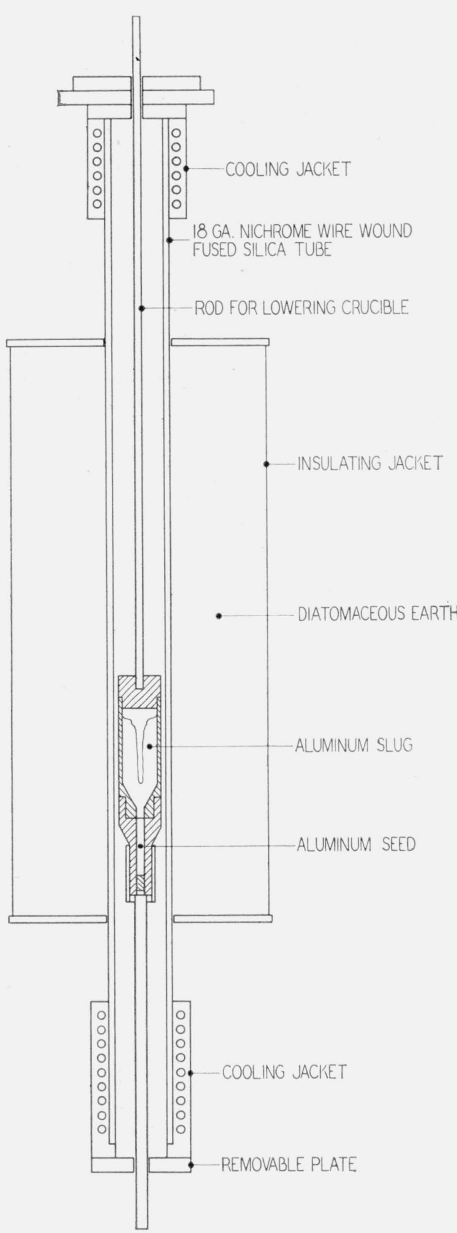

FIGURE 1. Diagrammatic sketch of vertical crystal-growing furnace showing "seeding" device.

Approximately one-tenth size.
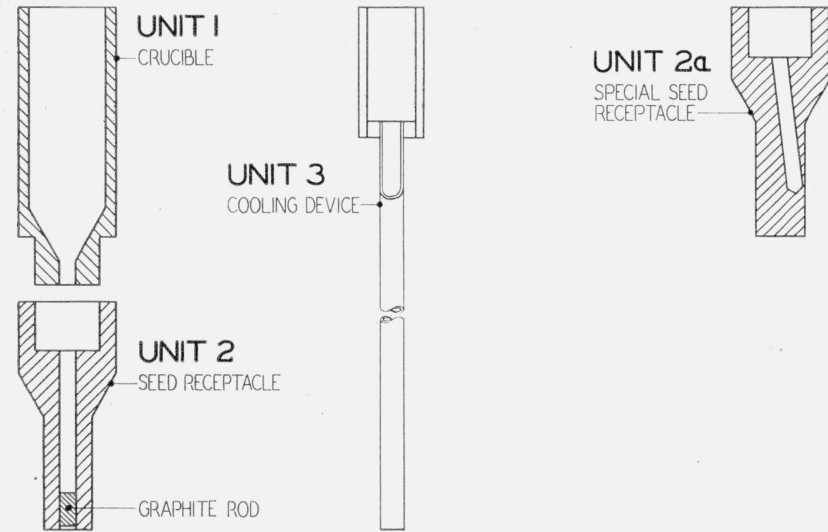

Figure 2. Longitudinal sections of crystal crucible, "seed" crucibles, and cooling device.

Approximately one-fifth size. produced in multiple in a single crucible. The axial orientation of the seed crystals is obtained by X-ray diffraction methods.

The following procedure is used to assemble the apparatus prior to the growing of preferentially oriented single crystals of aluminum.

(1) The charge is melted and allowed to solidify in the crucible shown in unit 1 , figure 2 , its bottom having been fitted with a tightly fitting graphite cap in order to produce a flat surface on the bottom of the ingot.

(2) The seed is put into unit 2, and units 1 and 2 are put together.

(3) By means of a tightly fitted graphite rod, the seed is pushed up until it is seated against the lower surface of the ingot in unit 1 . Units 1 and 2 are both machined from graphite.

(4) The bottom of the seed receptacle is then placed in unit 3 , the cooling device, the seed receptacle bottom having been machined so that it will fit snugly into unit 3 . The receptacle of the cooling device is of copper, nickel-plated, and the long tube is of aluminum. The ring used to connect the nickelplated copper receptacle to the aluminum tube is of copper, tightly fitted into the receptacle, the aluminum tube in turn being tightly fitted into the connecting ring. The aluminum tube should be of such length so that it extends out into the atmosphere, as shown in figure 1.

The object of the cooling device is to conduct just enough heat away from the bottom of the seed receptacle so that only the upper portion of the seed will melt. Then, upon slowly lowering the entire assembly in the furnace, the melted portion of the seed and the melt in unit 1 will freeze and assume the orientation of the unmelted portion of the seed.

The correct furnace temperature to permit just partial melting of the seed is obtained by trial, using a thermocouple in the immediate vicinity of the receptacle of the cooling device to obtain the correct temperature for a specified location of the entire assembly in the furnace. It is suggested that seeds of undesirable axial orientation be used in the determination of the correct furnace temperature, because, until this temperature is established for a fixed position of the assembly in the furnace, there is a possibility that the entire seed might be melted. This temperature can usually be established after a few trial runs. Once this temperature has been fixed, the same percentage of seed can be melted on each subsequent run, provided the assembly is placed in the same relative position in the furnace each time. Seed crystals melted in this manner may be used over and over again.

It is possible that many seed crystals may be grown without obtaining any having the exact axial orientation desired. It is possible, however, to obtain seeds having axial orientations within several degrees of being parallel to the desired orientation. Such seeds can be used for growing preferentially oriented single crystals by the proper placing of the seed in a seed receptacle that has its cavity at the same angle with the axis of the seed receptacle as the axis of the seed makes with the 


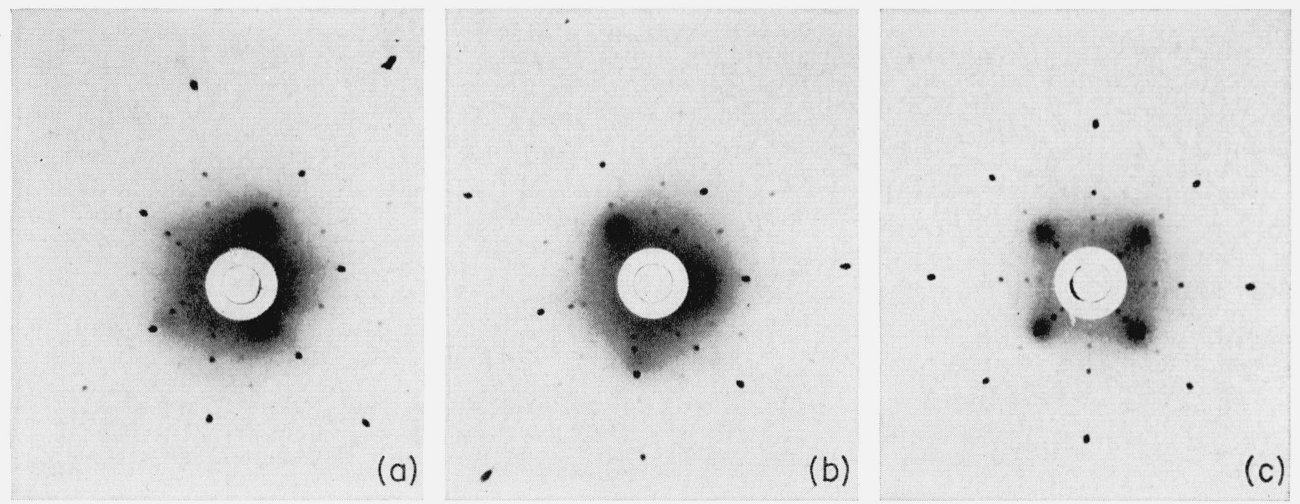

FIGURE 3.-Laue back-reflection diffraction patterns showing axial orientations.

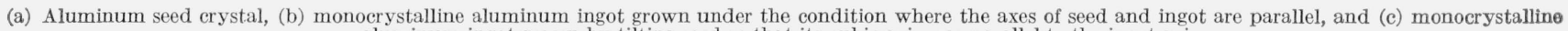
aluminum ingot grown by tilting seed so that its cubic axis was parallel to the ingot axis.

orientation desired in the axis of the crystal to be grown. A sketch of such a seed receptacle is shown in unit $2 \mathrm{a}$, figure 2 . When a seed receptacle of this type is used, the top of the seed should be ground so that its surface is in the same plane as the bottom of the recess in the seed receptacle.

Figure 3 is a photograph of Laue back-reflection diffraction patterns obtained from an aluminum seed crystal and two monocrystalline aluminum ingots produced from the same seed crystal, the incident X-ray beam having been parallel to the axes of the seed and ingots. The patterns are typical of the accuracy attainable in the abovedescribed method for growing preferentially oriented aluminum monocrystals.

The author expresses his appreciation to W. F. Gerhold for assistance in establishing the abovedescribed method.

Washington, November 20, 1957. 